\title{
Influenza surveillance: experiences from establishing a sentinel surveillance system in Germany
}

\author{
Joachim Szecsenyi, Helmut Uphoff, Sigrid Ley, Hans Dieter Brede
}

\begin{abstract}
Objectives - Before and during peak influenza periods there is increased morbidity from other respiratory tract disorders. Sentinel networks of primary care physicians can be very effective in the early detection of influenza epidemics and the German network, the Arbeitsgemeinschaft Influenza (AGI), began its work in this area in 1992.

Methods - Data are transmitted weekly from the doctor's computer via Btx to a central computer. The numerator is the weekly number of acute respiratory infections (ARI) in five age groups and the denominator is the weekly number of patient consultations. Data on hospitalisation, mortality, and days of sick leave from work or school are also collected. Swabs for influenza specimens are collected in 30 physicians' offices each Monday and sent to three reference centres.

Findings - During the last recording period, from week 461993 to week 151994 , 411 physicians' offices participated in the network. For 16 to 22 weeks, more than $60 \%$ of the participants transmitted data. During both the 1992-93 and 1993-94 influenza seasons, peaks were observed in the rate of ARI. There was a corresponding increase in sick leave from work and school. Rates for hospitalisation and deaths due to influenza showed no peaks during either season.

Conclusions - Although the German sentinel network for influenza experienced some technical problems in the first year, it was possible to solve these. Reporting rates were very satisfactory in the second year. The network will now be expanded to include 750 physicians in order to receive 600 weekly reports and obtain a solid baseline for an early warning system.
\end{abstract}

(F Epidemiol Community Health 1995;49 (Suppl 1):9-13)

Mortality associated with influenza is considerable, especially in high risk groups such as the elderly. Before and during peak influenza periods there is increased morbidity from respiratory tract infections. ${ }^{1-3}$ An increase in the frequency and severity of asthma attacks has also been reported. ${ }^{4}$ During epidemics of influenza viruses $A$ and $B$ excess mortality from these can be distinguished from other causes, such as respiratory syncytial virus (RSV), which are more consistent in their occurrence. ${ }^{5}$ In many countries, sentinel networks of primary care physicians have shown that these can be a very effective tool for the early detection of influenza epidemics by means of continuous reports of morbidity data on acute respiratory tract disease..$^{6-8}$ In addition, specimens for virus isolation and immunoassay can be obtained from these sources. Direct benefits of the early detection of the spreading of influenza, the initiation of further control measures and amantadine prophylaxis and vaccination campaigns for high risk groups, are outlined and discussed. ${ }^{9}$ Even in non-epidemic years, the impact of influenza on morbidity and mortality can be high. ${ }^{10}$

Sentinel surveillance of influenza, based on doctors' practices, could also play a part in increasing influenza vaccination rates in general and, in particular, in high risk populations. ${ }^{11}$ Until 1990, there was a well established monitoring system for influenza in the former German Democratic Republic. ${ }^{12}$ This monitoring system was mainly based on a central register with data obtained from laboratories, pathologists, health centres, and i arosurveillance studies. It had to close down shortly after the reunification with West Germany because of the adoption of the West German health care system and legislation. In the field of ambulatory care there are more specialists than GPs practising privately. In urban and suburban areas, in particular, children are more likely to be treated by paediatricians, who are commonly regarded as belonging to the primary care sector. Primary care physicians in Germany see a larger number of patients per day than their counterparts in other European countries, partly due to the fee for service basis of remuneration. ${ }^{13}$

There are difficulties associated with setting up networks such as these. Firstly, there is no tradition or positive attitude towards participating in primary care research activities in Germany, which makes it very difficult to establish projects. Secondly, because primary care physicians do not have fixed patients lists, a population based denominator can only be estimated. ${ }^{14}$ Last, but not least, government financial support for epidemiological research is very small. The attempt to establish a sentinel surveillance system for influenza had to take these special circumstances into account beforehand.

In 1992, the Arbeitsgemeinschaft Influenza (AGI) was founded to establish a nationwide 
physician's office-based influenza surveillance system. The AGI has a scientific board whose members are made up of cirologists, epidemiologists, general practitioners, paediatricians, and representatives from scientific and professional organisations and health authorities. Since there is no government support the project is sponsored in total by the four suppliers of influenza vaccine operating in Germany. The sentinel network of the AGI hopes to include 750 physicians in their work in the 1994-95 influenza season.

\section{Methods}

ORGANISATION OF THE NETWORK

The organisation and management of the entire network (fig 1) is carried out by Medialog, a private company with long experience in the public health care sector. Central data collection and statistics are carried out by Anfomed, a company equipped with sufficient computer capacity and knowledge. Virus isolation is conducted by three different laboratories (George-Speyer-Haus in Frankfurt, WHO National Collaborating Centre for Influenza-Surveillance in Hannover, and the Institute of Virology at the University of
Marburg). All participating doctors volunteered after an appeal was published in medical journals or was made by direct mail or by professional organisations. A stepwise expansion from 200 physicians' offices in the first year to 400 physicians' offices in the second year was targeted to keep the whole project manageable.

DATA TRANSFER FROM THE PHYSICIAN'S OFFICE

All computerised physician's offices are supplied with a modem and terminal software. The data transfer is conducted via Btx, a standardised service provided by the German telephone company, Telecom. It allows all of the physicians' offices to be connected simultaneously through the Btx network. A telephone hotline was established for technical support. Physicians without a computer received prestamped envelopes for mailing their data, which are then entered in the file. Data are recorded from week 46 to week 15 in the following year. Physicians' offices which had already established transfer systems were asked to $\log$ into the Btx system from the beginning of week 44 , allowing them to receive information about the coming recording season as well as "warming up" the whole network.

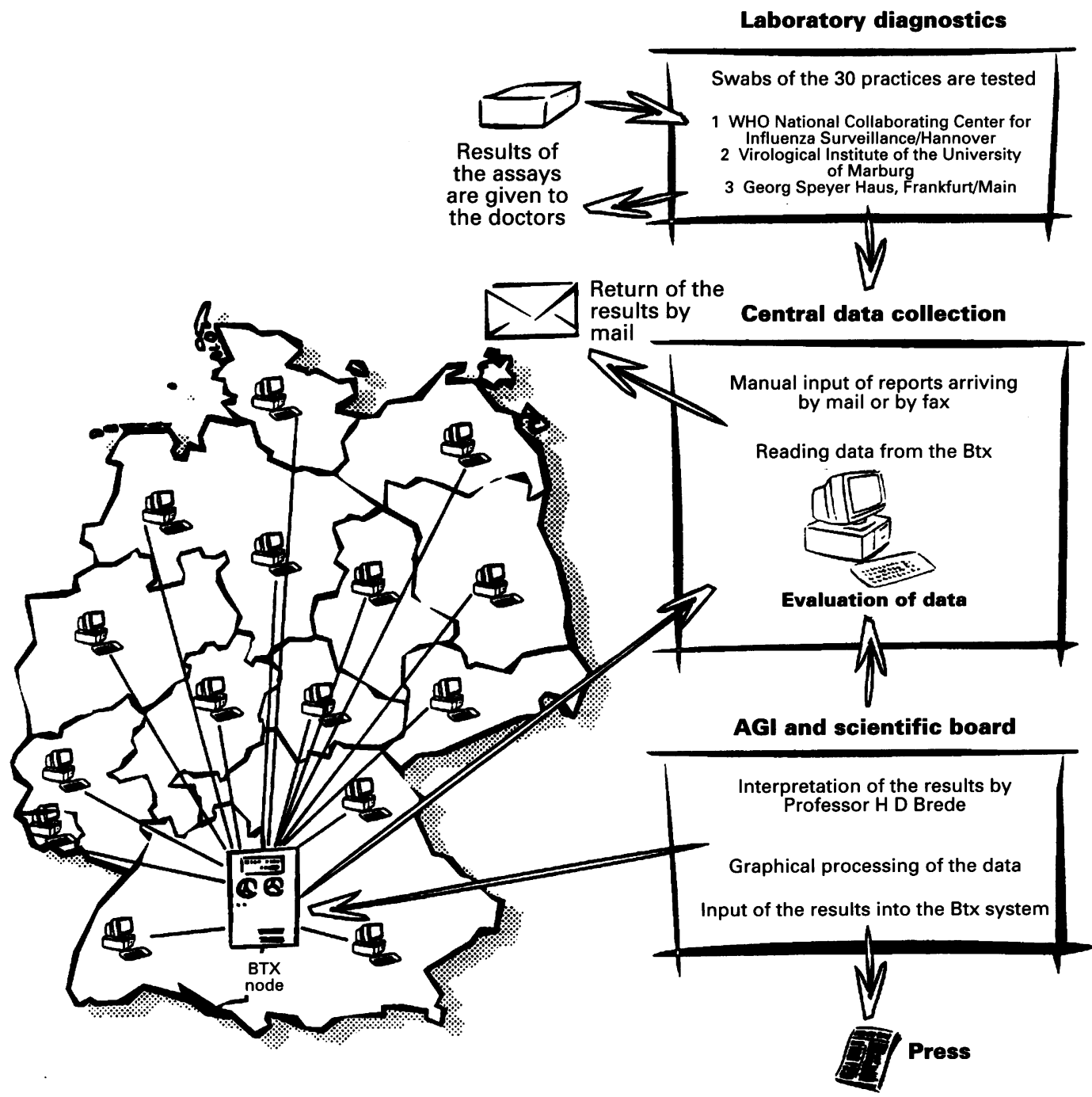

Figure 1 Data collection, evaluation, and return of the results. 


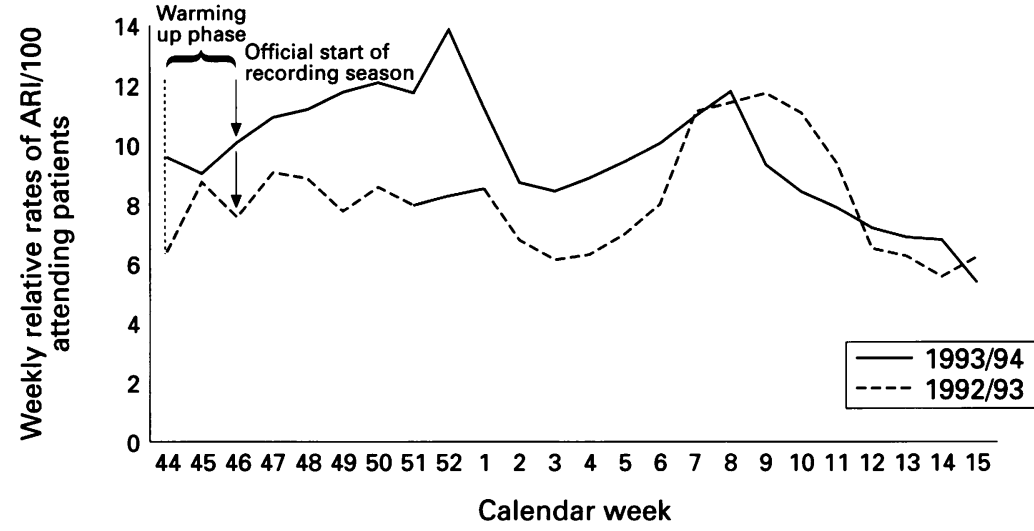

Figure 2 Weekly rates of acute respiratory infections (ARI) during the recording seasons 1992-93 and 1993-94.
VALIDITY OF DATA ON MORBIDITY

According to a recommendation made by Snacken et $a l,{ }^{6}$ we use all ARI as an indicator. Participating physicians attended one of the half day meetings that were held all over the country. During these meetings, criteria were discussed extensively for determining the cases to be included in the database. These discussions aimed to make physicians aware of interphysician variations and to make explicit the individual criteria used by each doctor. Taking into account the fact that there will always be some interpractice and interphysician variations, ${ }^{16}$ we asked the doctors to reconsider their criteria, but then after doing so to remain as consistent as possible within their individual pattern of recording once they had started to work with the network. Knowing well that sentinel networks can never measure true morbidity or incidence rates because of over or under reporting, we wanted to reduce bias in the trends by taking these measures.

Thirty randomly chosen physician's offices send swabs taken on each Monday from the first five patients suffering from ARI. Each laboratory receives the swabs from 10 different physician's offices. At the WHO National Collaboration Centre for Influenza Surveillance in Hannover, the agents are cultured to detect the exact types and subtypes of the influenza viruses. A new ELISA, capable of detecting influenza A and B, RSV, parainfluenza, and Myocoplasma pneumoniae is being used at the Georg-Speyer-Haus. ${ }^{15}$ The Institute of Virology at the University of Marburg uses both methods for purposes of comparison.

DATA INTERPRETATION AND FEEDBACK

All of the collected information is gathered at the AGI until Thursday each week. The data are then evaluated and commented on in cooperation with HD Brede. After having been processed graphically, an evaluation of the weekly epidemiological situation is transmitted back to the participating physicians' offices via $B t x$ or letter, whichever is applicable. The health authorities and media are informed by fax.

\section{COLLECTED DATA}

On the basis of experience gained in other sentinel systems, it should be possible to obtain the data collected by the practitioners with little effort, while at the same time yielding maximum information. The registered events should exhibit a frequent occurrence over the entire registration period. Our registration form, either printed or displayed by $\mathrm{Btx}$ on the screen of the computers in the physician's offices, requests the physician to report the first contact with all patients suffering from ARI. This number is recorded daily in five age groups $(0-5,6-15,16-34,35-60$, more than 60 years of age). The number of sick leaves with respect to work or school, hospitalisation, and deaths caused by ARI are registered per week. As a denominator, we use the weekly number of patients who visit the physician's offices.

\section{Findings}

Beginning with 182 physicians' offices during the 1992-93 influenza season, the network grew to a total number of 411 during the 1993-94 seasons. Twenty nine $(7 \%)$ of these were the offices of paediatricians and 352 $(86 \%)$ were those of general practitioners. The highest reporting rate was reached in week 6 of 1994, when 300 physicians' offices $(72.9 \%)$ transmitted their data. The lowest reporting rate $(47 \cdot 7 \%)$ occurred in week 52 , a traditional vacation period in Germany due to Christmas and New Year's Eve. Nevertheless, reporting rates were over $70 \%$ during 6 out of $22(27 \cdot 3 \%)$ weeks and between 60 and $70 \%$ during another $10(45.5 \%)$ weeks of the season.

The ratio used for the evaluation - number of ARI per 100 attending patients - has turned out to give the best baseline and to be the most sensitive ratio to changes in morbidity as reported from other sources. Comparing baseline data from general practitioners with data from paediatricians, we found a significantly higher relative rate of ARI reported by paediatricians. On average, an excess in the baseline of $10-12 \%$ was observed. We made similar observations in smaller practices with less than 200 attending patients per week $(n=$ 71 practices).

Using data from all the participating physicians' offices, we observed a relatively smooth increase in the baseline in both the 1992-93 and the 1993-94 seasons. In 1992-93 it started with a rate of $7 \cdot 5$, reached a small peak in week 52 , and then a second peak between weeks 7 and 10 in 1993. Similar observations occurred in the 1993-94 season at a higher baseline level (fig 2).

The weekly number of sickness certificates that were sent to schools or places of work once again showed a higher baseline for the 1993-94 season, with the exception of the fact of a higher peak in weeks 7 and 10 in both seasons in 1993-94 (fig 3).

Reported rates of hospitalisation due to ARI were low for both seasons: they ranged between 


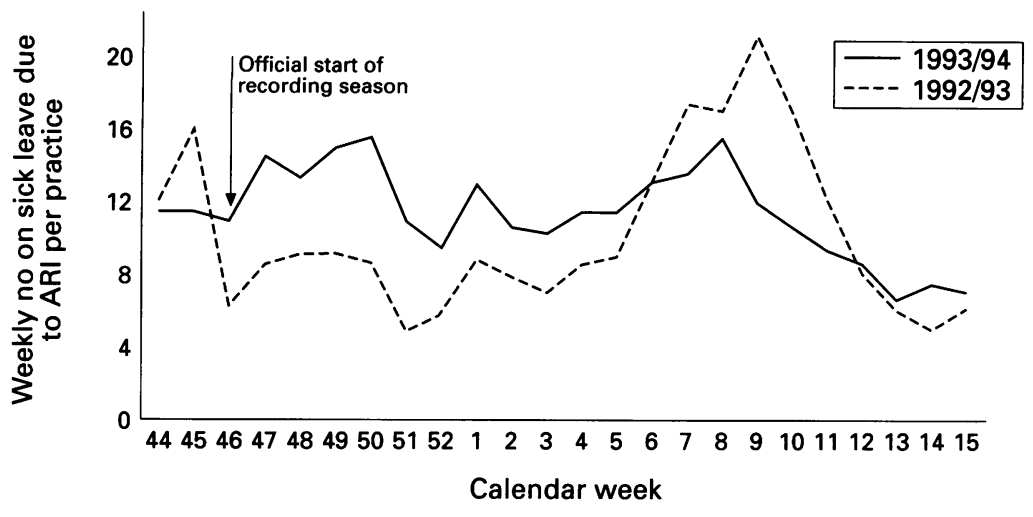

Figure 3 Weekly absolute numbers of sick leave from school or work due to acute respiratory infections (ARIs) during the recording seasons 1992-93 and 1993-94.
0.00 and 0.14 per physician's office and week. No significant peaks could be observed. Reported weekly death rates because of influenza or ARI were close to zero during both seasons. A total of 15 deaths were reported during the entire 1992-93 season, but the corresponding number for the 1993-94 season amounted to 65.

The ELISA we used for diagnostic purposes showed some practical disadvantages. If the physician's office did not send the swabs regularly, they had to be stored in order to reduce wasting the coated ELISA plates. After transportation and storage of the swabs, the assay showed distinct sensitivity to the antigens. The ratio of influenza $\mathrm{A}$ assays correlated to other observations. The ratio of RSV positive swabs was high (75\%). Mycoplasma and parainfluenza could not be detected, with the exemption of the control specimens.

\section{Discussion}

We were able to achieve our aim of involving 400 primary care physicians' offices well distributed throughout Germany in the sentinel network within a two year period. Although the network is still expanding and the practitioners have only seldom participated in research, the reporting rates were higher than those in well established projects in other countries. ${ }^{1718}$ The number of participating physicians' offices may be increased to 750 in the future in order to receive 600 weekly reports.

Data transfer and feedback between the physicians' offices and AGI proved to be effective, although there were numerous technical problems that had to be solved in the first year. Sending data by mail is less common as the use of patient held insurance cards is now forcing more physicians' offices to computerise. In 1994, we extending the period of recording from week 40 to week 15 in 1995 in order to obtain better baseline data.

Data from this new network still have to be interpreted carefully. It is not clear, for example, whether the rise in the baseline between 1992-93 and 1993-94 was due to an increase in actual morbidity or to other reasons. The selection of new physicians' offices could have biased this data as could changes or differences in the perception and case finding of ARI by participating practitioners. We tried to reduce such bias by discussing the criteria for including cases in small group sessions with the participating physicians at the beginning of the study. Furthermore, we are planning regular meetings as well as the use of video material demonstrating case samples to reduce such variability.

During the registration period 1993-94, influenza activity was very moderate in Germany. Considerable variation between the Bundesländer (the German federal states) became obvious. Only in the western parts of Germany, from Schleswig-Holstein in the very north down to Baden-Württemberg, did the ratio of ARI exceed the normal level. This took place in week 50 in 1993, when many western European countries reported a peak in influenza related morbidity. ${ }^{19}$ The same observation was made in week 8 of 1994, when influenza morbidity was highest in many eastern European countries. Both peaks were moderate in Germany, and the ratio of ARI per 100 physicians' offices did not exceed $17 \%$ in any region. The average for the whole country was $12 \cdot 1 \%$ in week 50 and $11.8 \%$ in week 8 . These observations matched our laboratory results in which influenza could first be assayed in the 45 th week. The incidence of cultured influenza virus and the rate of serum positive probes in local laboratories remained low. According to our observations, RSV and $M$ pneumonia, in particular, showed significant activity.

The observations during the last influenza season showed Germany as a buffer zone between eastern and western European countries. Surprisingly, both peaks in morbidity caused by ARI occurred in the western part of Germany. This might be partly explained by the fact that influenza A-H3N2, the predominant subtype, had already circulated in the eastern part of the country a year earlier.

The ELISA, used as a quick and easy assay for agents causing ARI, showed some disadvantages that have to be seen in connection with organisational problems. The swabs were sometimes not evaluated immediately at the laboratory, but stored in order to wait for delayed incoming specimens. The organisation in the cooperating laboratories has to be improved as has the buffer used as storage medium during the often prolonged transportation by mail.

Our period of recording was six weeks shorter than in most other European networks, mainly because of reasons of feasibility for the physicians and cost containment. In 1994 we expanded this period from week 46 to week 15 in 1995.

The authors would like to thank the practitioners and their staff for their active participation. We are also grateful to $\mathrm{Dr}$ Willers from the WHO National Collaborating Centre for Influenza in Hannover and Prof Dr Slenczka from the University of Marburg for their virological diagnostic tests.

1 Fleming DM, Cross KW, Crombie DL, Lancashire RI. Respiratory illness and mortality in England and Wales. A study of the relationships between weekly data for the incidence of respiratory disease presenting to general practitioners, and registered deaths. Eur f Epidemiol 1993 9:571-6.

2 Conolly AM, Salmon RL, Lervy B, Williams DH. What are 
the complications of influenza and can they be prevented? Experience from the 1989 epidemic of $\mathrm{H} 3 \mathrm{~N} 2$ influenza $A$ in general practice. $B M F$ 1993;306:1452-4

3 Fleming DM, Ayres JG. Diagnosis and patterns of incidence of influenza, influenza-like illness and the common cold in general practice. $\mathcal{F} R$ Coll Gen Pract 1988;38:159-162. 4 Bollag U. Asthmaanfälle in der Praxis - epidemiologische Merkmale und saisonale Muster in der Longitudinal beobachtung. Gesundh-Wes 1993;55 (suppl 1):42-5.

5 Fleming DM, Cross KW. Respiratory syncytial virus or influenza? Lancet 1993;342:1507-10.

6 Snacken $R$, Lion J, van Casteren V, et al. Five years of sentinel surveillance of acute respiratory infections (19851990): the benefits of an infleunza early warning system. Eur 7 Epidemiol 1992;8:485-90.

7 Fleming DM. The measurement of morbidity in general practice. $\mathcal{f}$ Epidemiol Community Health 1991;45:180-3.

8 Costagliola D, Flahault A, Galinec D, Garnerin P, Menares Costagliola D, Flahault A, Galinec D, Garnerin P, Menares
J, Valleron A-J. A routine tool for detection and assessment of epidemics of influenza-like syndromes in France. $\mathrm{Am}$

f Public Health 1991;81:97-9.
9 McBean AM, Babish JD, Warren JL. The impact and cost of influenza in the elderly. Arch Intern Med 1993;153: 2105-11.

10 McDonald CJ, Hui SJ, Tierney WM. Effects of computer reminders for influenza vaccination on morbidity during influenza epidemics. MD Computing 1992;9:304-12.

11 Van Essen GA. Call-up for influenza vaccination of all patients aged $65+$. A study of turnout, motivation and awareness in three general practices. Huisarts Wetenschap 1992;35:345-9.

12 Glathe $H$, Rasch G. Influenzaüberwachung in der ehemaligen DDR im Zeitraum 1969-1990. Bundesgesundheitsblatt 1992;35:500-6.

13 Boerma WGW, de Jong FAJM, Mulder PH eds. Health car and general practice across Europe. Utrecht: Nivel, 1993.

14 Szecsenyi J, Engelhard N, Wessel $M$, et al. Eine Methode zur Bestimmung des Denominators in Allgemeinpraxen Ergebnisse einer Pilotstudie. (A method to determine the denominator in general practice - results from a pilo study.) Das Gesundheitswesen 1993;55 (suppl 1):32-6.

15 Döller G, Schuy W, Tjhen KY, Stekeler B, Gerth H-J. Direct detection of influenza virus antigen in nasophyaryngeal specimens by direct enzyme immunoassay in comparison with quantitating virus shedding. $尹$ Clin Microbiol 1992; with quan

16 Crombie DL, Cross KW, Fleming DM. The problem of diagnostic variability in general practice. $f$ Epidemiol Comdiagnostic variability in general

17 Buffington J, Chapman LE, Schmelz LM, Kendal AP. Do family physicians make good sentinels for influenza? Archives of Family Medicine 1993;2:859-865.

18 Chauvin P, Diaz C, Garnerin P, et al. Surveillance en France. Bull Épidemiol Hebdomadaire 1993;21:93-6.

19 Institut Pasteur. EUROGROG weekly bulletins. Paris: Institut Pasteur, 1993. 